\title{
Une approche d'enseignement des stratégies de compréhension de lecture de textes courants auprès de jeunes lecteurs à risque d'échouer
}

\author{
CATHERINE TURCOTTE \\ Université du Québec à Montréal \\ MARIE-HÉLÈNE GIGUÈRE \\ Université de Montréal \\ MARIE-JULIE GODBOUT \\ Université du Québec à Montréal
}

\section{Résumé}

Ce projet de recherche mené en milieu défavorisé a impliqué 20 enseignants de la $4^{\mathrm{e}}$ à la $6^{\text {e }}$ année du primaire. Ces derniers se sont engagés à enseigner des stratégies de compréhension de lecture de façon explicite aux élèves à partir de textes courants venant de diverses sources. En septembre 2012 et en avril 2013, leurs élèves ( $n=273)$ ainsi que des élèves venant de classes ne recevant pas l'intervention $(\mathrm{n}=101)$ ont répondu à une épreuve de compréhension de lecture. Les résultats suggèrent que les élèves des classes avec intervention, beaucoup plus faibles en début d'année, rattrapent l'écart qui les sépare des élèves des classes sans intervention en fin d'année.

Les lecteurs compétents utilisent une grande variété de connaissances, d'habiletés et de stratégies de lecture (Pressley, 2006; Turcotte \& Cloutier, 2014). Ils arrivent d'ailleurs à adapter leurs stratégies et à les contrôler adéquatement en fonction des caractéristiques du texte (Meyer \& Ray, 2011). Ces lecteurs atteignent un niveau de littératie élevé, ce qui leur permet de traiter de l'information écrite complexe et exigeante pour comprendre et apprendre (Conseil Canadien sur l'Apprentissage, 2008). Pour en arriver là, ils ont dû bien évidemment apprendre à identifier correctement et rapidement les mots, mais ils ont également appris à comprendre les textes écrits en s'appuyant sur leurs connaissances des structures de textes diverses (Dymock, 2005), du vocabulaire technique, scientifique et disciplinaire (Woolley, 2011), d'éléments particuliers tels les figures, les graphiques, les intertitres (Donovan \& Smolkin, 2006), etc. Au $21^{\mathrm{e}}$ siècle, ces lecteurs compétents ont également appris à adapter leurs stratégies d'identification, d'auto-questionnement et de synthèse des informations importantes pour lire des textes numériques (Leu, Kinzer, Coiro, Castek, \& Henry, 2013). Les écrits imprimés et numériques, les types de textes et les sujets traités sont ainsi plus que jamais variés.

Des modèles illustrant la compréhension en lecture (Irwin, 2006; Perfetti, Landi \& Oakhill, 2005) expliquent d'ailleurs que cette dernière se construit continuellement grâce à l'interaction entre diverses habiletés et stratégies, elles-mêmes se référant à plusieurs unités de langage. Selon le modèle des processus de lecture d'Irwin (2006), les lecteurs doivent 1) comprendre chaque phrase, 2) relier les phrases entre elles, 3) comprendre la 
structure du texte et 4) élaborer leur compréhension à partir de connaissances et d'expériences personnelles. Toujours selon Irwin, la compréhension en lecture ne peut être une activité passive, ou encore le résultat d'une liste statique de sous-habiletés. Ces processus doivent être pilotés et contrôlés par des stratégies cognitives et métacognitives. Pour arriver à une compréhension adéquate, de nombreux processus cognitifs interagissent avec les connaissances du lecteur et ses stratégies de contrôle pour construire une représentation mentale du texte (Van Dijk \& Kintsch, 1983). La compréhension écrite est donc complexe, multidimensionnelle et en développement continuel (Paris, 2005; Perfetti, Landi \& Oakhill, 2005).

\section{Problématique}

Alors que les exigences en littératie ne cessent d'augmenter et que notre connaissance de la complexité de la compréhension se raffine, les pratiques d'enseignement de la compréhension en lecture semblent toujours inadéquates pour une bonne partie des élèves du primaire. Au Québec, en sixième année, 74,7\% des élèves réussissent l'épreuve ministérielle obligatoire en lecture. Ce taux chute à 58,6\% lorsque les élèves proviennent d'un milieu défavorisé (Desrosiers \& Tétreault, 2012). Les lecteurs de la sixième année, surtout en milieux défavorisés, sont donc loin de tous atteindre les compétences attendues avant d'entrer en première secondaire. D'ailleurs, au secondaire, les exigences en matière de compréhension qu'imposent les textes disciplinaires sont fréquemment plus élevées que les compétences des élèves (Shanahan \& Shanahan, 2008). Ainsi, tout au long du primaire, pour réussir leur scolarité, les élèves ont besoin de développer leur vocabulaire, d'être exposés à des textes de complexités variables sur une grande diversité de sujets venant de plusieurs sources, en plus de développer des stratégies de compréhension de lecture à travers toutes les disciplines (Biancarosa \& Snow, 2004; Torgesen, Houston, Rissman, Decker, Roberts \& al., 2007; Watson, Gable, Gear \& Hughes, 2012).

Cet article présente les premiers résultats d'une étude dans laquelle se sont engagés une vingtaine d'enseignants de la 4e à la 6e année. Ces enseignants, provenant d'écoles reconnues comme étant les plus défavorisées d'une commission scolaire en banlieue de Montréal, ont été invités à enseigner des stratégies de compréhension de lecture de textes courants. L'intervention, de même que les instruments d'évaluation conçus spécifiquement pour le projet, se sont appuyés sur une large revue d'écrits scientifiques portant sur l'enseignement de stratégies de compréhension de textes courants et sur l'importance de ces apprentissages.

\section{L'importance de comprendre les textes courants}

Depuis quelques années, des chercheurs nord-américains (Bryce, 2011; Hall \& Sabey, 2007; Juel, Hebard, Park-Haubner \& Moran, 2010) ont mis de l'avant l'importance d'exposer les élèves aux textes courants dans différentes disciplines très tôt dans la scolarité afin de les familiariser avec des macrostructures, du vocabulaire et des thèmes variés. À ce propos, au fur et à mesure que les élèves vieillissent, la présence de ces textes dans le milieu scolaire et dans les loisirs augmente clairement. Selon Moss (2004), à partir de la $6^{\mathrm{e}}$ année, $75 \%$ des textes rencontrés par les élèves ne sont plus des textes narratifs. De plus, la pratique de la lecture sur Internet, composée en majorité de 
textes courants, fait partie intégrante des habitudes de lecture des jeunes (Hughes-Hassell \& Rodge, 2007; Kamil \& Lane, 1998). L'utilisation et la compréhension des textes courants sont donc cruciales pour réussir sa vie scolaire, professionnelle et sociale (Duke, 2000).

Néanmoins, les textes préférés des enseignants des premières années du primaire reflètent peu ce besoin de se familiariser avec une grande variété de textes (Ness, 2011). Une étude de Duke réalisée en 2000 sonne l'alarme en révélant que les textes informatifs en classe de $1^{\text {re }}$ année sont d'une incroyable rareté. En effet, les 20 classes observées dans le cadre de sa recherche ont montré une utilisation moyenne de textes informatifs à raison de seulement 3,6 minutes par jour. Les classes provenant de milieux les plus défavorisés affichent pour leur part une moyenne accablante de 1,9 minute par jour. En 2011, l'équipe de Pentimonti, Zucker, Justice \& Kaderavek (2010) a analysé les quelques 426 livres lus par 13 enseignants du préscolaire durant une année scolaire complète. Ces chercheurs concluent que les textes narratifs sont hautement dominants et que les textes courants, les abécédaires et les livres offrant un contenu multiculturel sont largement minoritaires. Enfin, Martel, Lévesque et Aubin-Hort (2012) ont soumis un questionnaire portant sur les pratiques d'enseignement de la lecture à 413 enseignants québécois auprès d'élèves de 9 à 12 ans. Les auteurs concluent que le développement de la compétence à acquérir des connaissances à partir des textes est visé de manière modérée par la moitié des enseignants à l'étude.

Ces différentes études soulèvent un problème de taille. Non seulement il est difficile pour les élèves de développer leurs connaissances de divers types de textes quand ils ne sont pas en contact avec ces derniers (Gersten, Fuchs, Williams \& Baker, 2001), mais ce manque d'exposition aux textes courants entraine un enseignement minimal de la lecture centrée sur la maitrise de ces textes. Les élèves qui ne développent pas leur autonomie en lecture envers ces textes ont donc peu de chance de réussir au secondaire, là où la compréhension de textes courants est cruciale dans toutes les disciplines (Saenz \& Fuchs, 2002; Wigent, 2013).

Ce problème est d'autant plus inquiétant dans les milieux scolaires et familiaux défavorisés, souvent reconnus pour offrir moins de stimulation sur le plan du vocabulaire (Hart et Risley, 2004), du matériel écrit de qualité (Orr, 2003; Aikens \& Barbarin, 2008) et d'expériences variées sur le plan de l'écrit (Coley, 2002). En vieillissant, les élèves issus de ces milieux risquent davantage d'éprouver des difficultés, spécifiquement lorsqu'ils rencontrent des textes dans diverses disciplines (Chall, Jacobs \& Baldwin, 1990; Snow, 2008), car ces derniers diffèrent de ceux avec lesquels ils seraient plus familiers. Il appartient donc en grande partie à l'école d'enseigner les structures de ces textes, les stratégies de compréhension s'y rapportant et d'offrir un environnement riche en écrits variés.

\section{L'enseignement de la compréhension du texte courant}

En 2007, Gajria, Jitendra, Sood et Sacks (2007) publient une synthèse des recherches portant sur l'efficacité de l'enseignement de la compréhension des textes courants auprès des jeunes lecteurs à risque d'échouer. Les conclusions de leur synthèse précisent que l'enseignement explicite des stratégies est l'intervention ayant le plus d'effets positifs sur la compréhension de ces élèves en lecture. Deux méta-analyses 
portant sur les interventions en compréhension de lecture auprès des élèves éprouvant des difficultés (Sencibaugh, 2007; Berkeley, Scruggs \& Mastropieri, 2010) ont également conclu que l'enseignement explicite et systématique détient un impact positif et statistiquement signifiant sur la compréhension en lecture. Enfin, Bissonnette, Richard, Gauthier et Bouchard (2010) ont réalisé une méga-analyse afin d'examiner les meilleures approches d'enseignement de la lecture. Cette méga-analyse synthétise 11 méta-analyses ayant préalablement examiné 362 recherches publiées entre 1963 et 2006, ces dernières impliquant au total plus de 30000 élèves. Les résultats de la méga-analyse soutiennent que l'enseignement structuré, directif et explicite est le plus approprié pour favoriser la réussite en lecture chez les élèves en difficulté.

Brièvement, cet enseignement explicite vise l'introduction d'une stratégie à la fois, et ce, de façon structurée. Chaque stratégie est enseignée d'abord en expliquant pourquoi, comment et quand l'utiliser. Ensuite, cette stratégie fait l'objet d'une modélisation par l'enseignant sous forme de pensée à voix haute afin de rendre son application claire pour les élèves et fournir un modèle adéquat. La troisième étape consiste à guider les élèves dans l'utilisation de cette stratégie à l'intérieur d'une tâche et de leur offrir des rétroactions immédiates. Enfin, l'enseignant les amène à exercer de façon autonome leur stratégie dans des contextes variés (Archer \& Hugues, 2011). Cette approche permet aux élèves plus forts de passer plus rapidement à l'étape du travail autonome alors que les autres élèves peuvent bénéficier de soutien supplémentaire (Juel \& Minden-Cup, 2000). Cet enseignement direct et structuré visant l'apprentissage de stratégies de compréhension se révèle donc plus efficace qu'un enseignement plus occasionnel ou moins structuré (Bråten \& Anmarkrud, 2013), surtout auprès des élèves plus faibles (Ritchey, Silverman, Montanaro, Speece \& Schatschneider, 2012).

Plusieurs approches ou programmes mettent donc de l'avant des pratiques d'enseignement basées sur l'enseignement explicite des stratégies de compréhension. Ces programmes visent l'apprentissage de certaines stratégies pour rendre le lecteur le plus autonome et polyvalent possible. En effet, plus d'une stratégie doit être explicitée, guidée et expérimentée de façon autonome sur une longue période de temps, car on ne peut assumer que tous les élèves apprennent à maitriser ces stratégies de façon accidentelle ou de façon implicite (Sweet \& Snow, 2003). Les approches rapportées dans les études suivantes illustrent ces propos.

Dès les années 1980, Palincsar et Brown (1985) ont incité les enseignants à promouvoir la modélisation et l'interaction entre élèves afin de favoriser le développement des quatre stratégies de compréhension suivantes : prédire le contenu du texte, questionner le texte, clarifier des extraits et résumer l'information. Dans cette approche nommée « enseignement réciproque », les élèves modélisent leurs stratégies en sous-groupes afin de les partager. Une revue de 19 études expérimentales sur cette approche conclut que cette dernière est davantage bénéfique lorsque les quatre stratégies sont d'abord enseignées de façon explicite par l'enseignant (Rosenshine \& Meister, 1993).

De plus, en Californie, une équipe (Cervetti, Pearson, Barber, Hiebert \& Bravo, 2007) a voulu intégrer l'enseignement des sciences, de la lecture et de l'écriture. Leur programme «Seeds of science roots of reading » comprend l'enseignement explicite des stratégies suivantes lors de lectures en sciences : anticiper, utiliser les indices du texte, 
identifier les idées principales, interpréter les informations visuelles, chercher des informations, faire une carte conceptuelle des idées du texte, identifier la structure du texte et faire des inférences. Les résultats d'une étude quasi-expérimentale (Goldschmidt, 2010) ont notamment démontré que les élèves participants au projet ont progressé deux fois plus en lecture que les élèves de groupes sans intervention, et ce, de façon significative. De surcroît, plus les élèves bénéficiant du programme s'amélioraient en lecture, plus ils obtenaient des résultats élevés en sciences.

Scharlach (2008) a également vérifié l'efficacité d'un programme durant 40 semaines qui comprenait l'enseignement explicite, la pratique guidée puis la pratique autonome de huit stratégies de compréhension: prédire, visualiser, faire des liens, questionner, identifier l'idée principale, résumer, confirmer les prédictions, critiquer. Les élèves ayant bénéficié de cet enseignement ont montré un progrès significativement plus élevé lors de tâches de compréhension de courts textes que ceux ayant reçu un enseignement typique basé sur le curriculum.

En somme, ces méta-analyses, méga-analyses et études présentées à titre d'illustration ont toutes révélé des bénéfices d'enseigner de façon explicite les stratégies de compréhension. Ces écrits ont inspiré la création de séquences d'activités d'enseignement des stratégies de compréhension de textes courants de façon explicite auprès de jeunes élèves de milieux défavorisés et sous-performants en lecture. À la demande des partenaires de recherche en milieu scolaire, l'approche devait aussi permettre aux enseignants de développer une meilleure connaissance des processus de compréhension, des stratégies de lecture et de la façon d'évaluer les élèves à ces égards. Dans cet article, les effets de l'approche d'enseignement sur les progrès des élèves en compréhension écrite de textes courants seront rapportés et discutés. Les effets sur les connaissances des enseignants et leurs compétences en évaluation feront l'objet d'articles subséquents.

\section{Déroulement de l'intervention}

Les stratégies choisies dans ce projet ont été discutées avec des conseillers pédagogiques de la commission scolaire afin de bien répondre aux besoins les plus importants des jeunes lecteurs du milieu. Les stratégies enseignées entre septembre et décembre étaient les suivantes : prédire le contenu du texte, activer ses connaissances antérieures, identifier la structure du texte, faire des inférences, se poser des questions en lisant. De janvier à avril, les stratégies étaient les suivantes : comprendre les mots de substitution, comprendre les mots nouveaux à l'aide du contexte et des indices morphologiques, identifier l'information principale explicite et implicite. Les stratégies ont été enseignées à raison d'une par semaine. Néanmoins, toutes les trois à quatre semaines, l'enseignant révisait les stratégies enseignées avant de poursuivre.

Les enseignants participants ont reçu, au début de l'année scolaire 2012, une formation sur l'enseignement explicite et les stratégies de lecture du projet. Ils ont également reçu un guide d'accompagnement comprenant des idées d'activités à partir de textes courants, guidant leur enseignement explicite et modélisé, pour ensuite soutenir les élèves dans une pratique guidée avant de les laisser travailler de façon autonome. Les textes étaient sélectionnés à partir de magazines, de sites Internet et de livres documentaires afin d'offrir des expériences de lecture authentiques (Hill, 2014). Ils 
abordaient des thèmes scientifiques ou sociaux qui soutenaient l'intérêt des élèves (par exemple une forêt sous-marine de 52000 ans, l'invention de la gomme à mâcher, les courses extrêmes, une imprimante à pizza, etc.). Entre 10 et 14 ans, les élèves apprécient les textes qui décrivent des expériences étonnantes (Guthrie \& Davis, 2003), qui proviennent de magazines ou de sites web s'adressant aux jeunes et traitant de thèmes actuels (Worthy, Moorman \& Turner, 1999). De tels textes suscitent l'engagement affectif et cognitif des jeunes lecteurs (McRae \& Guthrie, 2009). Les enseignants étaient encouragés parfois à les imprimer, mais ils étaient également incités à repérer certains textes sur internet et à les projeter sur un tableau numérique interactif. Chaque description d'activité comportait également des informations à l'égard de l'enseignant afin qu'il comprenne la pertinence de la stratégie de lecture et la situe dans un modèle de processus cognitifs (Irwin, 2006). Les enseignants ont ensuite participé à trois rencontres pendant l'année scolaire afin d'évaluer les progrès de leurs élèves, recevoir un suivi de formation et échanger sur leurs pratiques. Enfin, une orthopédagogue et une conseillère pédagogique étaient disponibles de façon hebdomadaire dans chaque école pour accompagner les enseignants qui désiraient les observer en train de modéliser une stratégie, enseigner en équipe ou qui souhaitaient se faire observer dans le but ensuite d'obtenir une rétroaction de leur part.

\section{Méthodologie}

Puisque cette étude vise à documenter la pertinence d'une approche, il est nécessaire de pouvoir comparer les progrès des élèves participants à ceux d'élèves venant de groupes sans intervention. Le contexte de la recherche ne permettait pas de procéder de façon aléatoire pour distribuer les classes à l'intérieur de deux conditions puisque le projet, dès le départ, était issu d'un partenariat avec des enseignants déjà prêts à intégrer l'approche proposée. Les classes sans intervention ont donc été sélectionnées dans une école ayant un profil socioéconomique semblable, mais étant géographiquement éloignée des classes participantes pour éviter toute contamination de l'intervention.

\section{Participants}

Les élèves participants proviennent de 20 classes regroupées dans trois écoles de la banlieue de Montréal. Au total, 110 élèves de $4^{\mathrm{e}}$ année, 89 élèves de $5^{\mathrm{e}}$ année et 74 élèves de $6^{\mathrm{e}}$ année ont participé au projet. Les enseignants participants ont, dès le début du projet, décrit un grand nombre de défis dans leur milieu : le manque de ressources humaines pour soutenir les élèves éprouvant une diversité de problèmes d'adaptation scolaire et sociale, les besoins générés par une grande défavorisation en contextes familial et scolaire, les difficultés à organiser des services à long terme, le grand roulement de personnel dans leurs écoles, le manque de stabilité dans les projets mis en place, le manque de temps pour sélectionner des textes imprimés et numériques adéquats, la difficulté d'arrimer l'enseignement entre les niveaux scolaires, etc.

De plus, 36 élèves de $4^{\mathrm{e}}$ année, 30 élèves de $5^{\mathrm{e}}$ année et 35 élèves de $6^{\mathrm{e}}$ année d'une quatrième école ayant un indice de défavorisation identique de 9 sur 10 (Ministère de l'éducation, du loisir et du sport, 2011) ont également été soumis aux évaluations, mais sans avoir reçu l'intervention. Cette école fut identifiée par une orthopédagogue comme étant un milieu de comparaison adéquat puisque les enseignants appuyaient leurs 
pratiques sur le contenu de manuels scolaires en français n'abordant pas l'enseignement explicite des stratégies de compréhension.

\section{Les épreuves de compréhension}

Des épreuves ont été conçues pour les élèves de chaque degré afin de pister leurs progrès entre le début et la fin de la séquence d'enseignement. Ces épreuves rendent compte de la complexité de la compréhension écrite, car elles comprennent des questions sur de nombreux processus de compréhension.

Ces épreuves comportaient des questions sur un texte abordant un phénomène scientifique ou social. Un certain contrôle portant sur différents critères a été exercé afin de fournir des textes ni trop complexes, ni trop faciles aux élèves de chaque degré. Ces critères étaient inspirés d'écrits sur le sujet (Fisher, Frey \& Lapp, 2012; Gunning, 2003; Mesnager, 2002). Le vocabulaire des textes était ainsi contrôlé afin de présenter un défi surmontable lors de la lecture. De plus, la longueur des phrases, le nombre de mots du texte ainsi que le nombre de mots par phrase ont été contrôlés afin que les épreuves soient graduées en difficulté entre la $4^{\mathrm{e}}$ et la $6^{\mathrm{e}}$ année. Ceci permettra, dans une étude subséquente, de tester les élèves à chaque année et d'observer les progrès de façon longitudinale.

Afin de contrôler le niveau de difficulté des textes, ils ont également été analysés à l'aide d'un logiciel de lisibilité nommé LISI (Mesnager, 2002; 2011), qui calcule la longueur des phrases et de la complexité du vocabulaire. Selon Mesnager (2011), l'analyse du texte soumis au logiciel LISI indique un degré de difficulté sur une échelle de 2 à 10. Les résultats sont pertinents pour des textes d'au moins 350 mots, destinés aux élèves de 8 à 12 ans. Le texte s'adressant aux élèves de la $4^{\mathrm{e}}$ année porte sur les tornades. Ce dernier est composé de 548 mots et comprend 40 phrases. Son indice LISI était de 4,9. Les élèves de la $5^{\mathrm{e}}$ année ont lu un texte sur la carie dentaire. Ce texte comprenait 588 mots répartis dans 43 phrases. Son indice LISI était de 5,2. Quant aux élèves de la $6^{\mathrm{e}}$ année, ils ont lu un texte sur le trafic d'animaux exotiques. Ce texte contenait 692 mots, 42 phrases et un indice LISI de 6,5.

Le questionnaire écrit lié à chacun de ces textes s'appuyait sur un modèle utilisant des processus cognitifs de la lecture (Irwin, 2006). Tel qu'abordé dans la problématique de cet article, les différentes composantes de ce modèle illustrent bien la complexité de la compréhension écrite et rendent possible l'identification des forces et des faiblesses en compréhension de mots, de phrases, de liens entre les phrases, de paragraphes ou de la structure du texte. Chaque question de l'épreuve comprenait un objectif précis, ce qui évitait d'avoir des questions uniquement centrées sur l'évaluation des capacités de repérage des élèves, par exemple. Le tableau 1 indique l'objectif derrière chaque question, ainsi que le processus s $\mathrm{s}^{6} \mathrm{y}$ référant. Un test pilote administré auprès de 30 élèves par degré scolaire dans un milieu moyennement favorisé non participant à la recherche a permis de réajuster les textes et les questions afin de les rendre plus clairs, de reformuler les questions imprécises et d'améliorer la lisibilité des textes.

Par la suite, l'administration du test en septembre à tous les participants de la recherche a permis de faire des analyses à l'aide de la théorie de la réponse aux items. À l'issue de ces analyses, il appert que les questions 1 et 2 sont plus faciles que les autres, ce qui était prévu et qui répond à l'objectif d'identifier les processus, même les plus 
simples, qui représentent des difficultés. Les courbes d'information des items permettent d'examiner également le niveau de difficulté de chaque question et des épreuves plus globalement. À la lumière de ces courbes, nous pouvons confirmer que les épreuves s'adressent aux élèves moyens de la classe et qu'elles permettent de cibler les élèves à risque d'éprouver des difficultés en compréhension.

\section{Tableau 1}

Objectifs et processus visés dans l'épreuve de compréhension de lecture

\begin{tabular}{lll}
\hline Question & Objectif & Processus (Irwin, 2006) \\
\hline $\mathbf{1}$ et $\mathbf{2}$ & $\begin{array}{l}\text { Repérer une information littérale } \\
\text { Identifier l'idée principale explicite }\end{array}$ & $\begin{array}{l}\text { Comprendre une phrase } \\
\text { Comprendre la structure du } \\
\text { texte }\end{array}$ \\
$\mathbf{4}$ & Identifier l'idée principale implicite & $\begin{array}{l}\text { Comprendre la structure du } \\
\text { texte }\end{array}$ \\
$\mathbf{5 a}$ et 5b & Comprendre un mot de substitution & $\begin{array}{l}\text { Comprendre les liens entre les } \\
\text { phrases à partir des anaphores }\end{array}$ \\
$\mathbf{6}$ et 7 & Inférer un lien causal & $\begin{array}{l}\text { Comprendre les liens implicites } \\
\text { entre les phrases }\end{array}$ \\
$\mathbf{8 a}$ et 8b & Comprendre un mot difficile & $\begin{array}{l}\text { Comprendre une phrase } \\
\text { Élaborer en faisant un lien avec } \\
\text { ses connaissances antérieures }\end{array}$ \\
$\mathbf{1 0}$ & Faire une inférence pragmatique & $\begin{array}{l}\text { Comprendre les liens implicites } \\
\text { entre les phrases }\end{array}$ \\
$\mathbf{1 1}$ & Inférer un lien logique & $\begin{array}{l}\text { Comprendre la structure du } \\
\text { texte }\end{array}$ \\
\hline
\end{tabular}

\section{Collecte de données}

Toutes les règles d'éthique et de déontologie de l'université d'attache des chercheurs ont été suivies avant d'entreprendre l'étude. En septembre et en avril, les chercheurs ont été accueillis dans chaque classe participante afin de soumettre les élèves aux épreuves de compréhension de lecture. Cette épreuve durait 60 minutes et il n'était pas permis d'utiliser le dictionnaire ou tout autre référentiel.

Les questionnaires ont été corrigés par les chercheurs, qui se sont également chargés de l'analyse des données. Les questions $1,2,3,4,5 \mathrm{a}, 5 \mathrm{~b}, 6,7,8 \mathrm{a}, 8 \mathrm{~b}, 9$ et 10 valaient un seul point chacune, mais la question 11 valait 4 points en $4^{\mathrm{e}}$ année, car elle 
demandait d'inscrire quatre éléments dans un schéma conceptuel. Les élèves pouvaient donc, à cette épreuve, cumuler un total de 16 points. Concernant les analyses de réussite par question, qui seront présentées dans le tableau 2 , les élèves de la $4^{\mathrm{e}}$ année ayant inscrit trois ou quatre éléments sur quatre dans le schéma conceptuel (question 11) ont obtenu un score de réussite à cette question.

Pour les épreuves de la $5^{\mathrm{e}}$ et de la $6^{\mathrm{e}}$ année, la dernière question valait six points, car six éléments étaient attendus dans leur schéma. Ces épreuves étaient sur 18 points. Pour les analyses portant sur les scores de réussite à chaque question, les élèves de la $5 \mathrm{e}$ année et de $6^{\mathrm{e}}$ année ayant inscrit entre quatre et six éléments sur six dans le schéma conceptuel à cette question 11 obtenaient un score de réussite à cette question.

Ainsi, des analyses descriptives ont permis de voir le taux de réussite à chaque question pour chaque groupe d'élèves. Une comparaison des moyennes a également été effectuée pour vérifier s'il y a un progrès entre l'épreuve de septembre et d'avril pour les groupes avec et sans intervention.

\section{Résultats}

Les taux de réussite pour chaque question sont compilés et décrits pour les groupes d'élèves de $4^{\mathrm{e}}, 5^{\mathrm{e}}$ et $6^{\mathrm{e}}$ année au prétest et au posttest. Ils sont également présentés dans le tableau 2. Les moyennes au prétest, au posttest ainsi que les moyennes de progrès sont ensuite décrites pour tous ces groupes d'élèves.

\section{Tableau 2}

Taux (\%) de réussite à chaque question de l'épreuve administrée en septembre 2012 (pré) et en avril 2013 (post) aux élèves de la $4^{\mathrm{e}}$ à la $6^{\mathrm{e}}$ année provenant des groupes avec et sans intervention

\begin{tabular}{|c|c|c|c|c|c|c|c|c|c|c|c|c|}
\hline \multirow{3}{*}{ 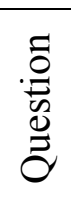 } & \multicolumn{6}{|c|}{ Élèves des groupes avec intervention } & \multicolumn{6}{|c|}{ Élèves des groupes sans intervention } \\
\hline & \multicolumn{2}{|c|}{$\begin{array}{l}4^{\mathrm{e}} \text { année } \\
(\mathrm{n}=110)\end{array}$} & \multicolumn{2}{|c|}{$\begin{array}{c}5^{\mathrm{e}} \text { année } \\
(\mathrm{n}=89)\end{array}$} & \multicolumn{2}{|c|}{$\begin{array}{c}6^{\mathrm{e}} \text { année } \\
(\mathrm{n}=74)\end{array}$} & \multicolumn{2}{|c|}{$\begin{array}{c}4^{\mathrm{e}} \text { année } \\
(\mathrm{n}=36)\end{array}$} & \multicolumn{2}{|c|}{$\begin{array}{c}5^{\mathrm{e}} \text { année } \\
(\mathrm{n}=30)\end{array}$} & \multicolumn{2}{|c|}{$\begin{array}{c}6^{\mathrm{e}} \text { année } \\
(\mathrm{n}=35)\end{array}$} \\
\hline & Pré & Post & Pré & Post & Pré & Post & Pré & Post & Pré & Post & Pré & Post \\
\hline 1 & 53 & 85 & 75 & 83 & 89 & 96 & 54 & 89 & 59 & 87 & 94 & 97 \\
\hline 2 & 51 & 64 & 74 & 86 & 86 & 91 & 56 & 64 & 94 & 81 & 92 & 91 \\
\hline 3 & 54 & 75 & 37 & 54 & 68 & 86 & 79 & 86 & 59 & 51 & 61 & 71 \\
\hline 4 & 24 & 51 & 20 & 25 & 68 & 51 & 82 & 72 & 56 & 35 & 58 & 57 \\
\hline $5 a$ & 41 & 82 & 60 & 83 & 76 & 95 & 77 & 83 & 31 & 84 & 58 & 97 \\
\hline $5 b$ & 28 & 66 & 19 & 33 & 67 & 89 & 48 & 72 & 31 & 48 & 86 & 89 \\
\hline 6 & 46 & 63 & 65 & 82 & 54 & 74 & 54 & 58 & 90 & 90 & 55 & 66 \\
\hline 7 & 50 & 66 & 62 & 52 & 43 & 73 & 59 & 75 & 59 & 61 & 77 & 77 \\
\hline $8 \mathrm{a}$ & 41 & 58 & 41 & 67 & 46 & 72 & 59 & 67 & 75 & 74 & 64 & 60 \\
\hline $8 b$ & 23 & 46 & 30 & 63 & 17 & 24 & 10 & 39 & 63 & 77 & 17 & 14 \\
\hline 9 & 75 & 89 & 25 & 71 & 31 & 95 & 87 & 94 & 81 & 80 & 89 & 91 \\
\hline 10 & 46 & 66 & 56 & 77 & 30 & 85 & 51 & 61 & 31 & 84 & 67 & 60 \\
\hline 11 & 32 & 74 & 50 & 68 & 9 & 43 & 56 & 55 & 65 & 68 & 56 & 54 \\
\hline
\end{tabular}


Les taux de réussite des élèves de la $4^{e}$ année du groupe avec intervention

En septembre, près de la moitié des élèves et parfois même un peu plus ont réussi les questions de repérage (questions 1 et 2), d'identification des idées principales explicites (question 3), de formulation d'inférences causales (questions 6 et 7) et logique (question 10).

Or, l'identification de l'idée principale implicite lors de la question 4 (24\% de réussite), la compréhension des mots de substitution en $5 \mathrm{a}$ et $5 \mathrm{~b}$ ( $41 \%$ et $28 \%$ ), les inférences lexicales $8 \mathrm{a}$ et $8 \mathrm{~b}(41 \%$ et $23 \%)$ et la compréhension de la structure du texte à la question $11(32 \%)$ sont réussies par peu d'élèves.

La question 9 visant la formulation d'une inférence pragmatique a été réussie par $75 \%$ des élèves. Elle le sera par une grande majorité $(89 \%)$ en fin d'année. En avril, toutes les questions étaient d'ailleurs réussies par davantage d'élèves. Une seule question est alors réussie par moins de la moitié des participants. L'inférence lexicale demandée (question $8 \mathrm{~b}$ ) est atteinte seulement par $46 \%$ des élèves en avril, mais ceci représentait deux fois plus d'élèves qu'en début d'année. Les questions 4 (51\%), 5a (82\%), 5b (66\%) et $11(74 \%)$ sont également réussies par au moins le double d'élèves si on compare avec le prétest.

\section{Les taux de réussite des élèves de la $5^{e}$ année du groupe avec intervention}

En septembre, plusieurs élèves de la $5^{\mathrm{e}}$ année ont pu repérer des informations littérales aux questions $1(75 \%)$ et $2(74 \%)$ dans le texte. Plus de la moitié des élèves ont inféré un lien de causalité aux questions 6 et 7 puis un lien logique à la question 10 . Enfin, 49\% des élèves comprenaient la structure du texte soumis (49\%) suffisamment pour remplir un schéma conceptuel adéquatement.

Or, moins de la moitié d'entre eux ont réussi à identifier l'idée principale explicite à la question $3(37 \%)$ et implicite à la question $4(20 \%)$ ainsi qu'à faire des inférences lexicales aux questions $8 \mathrm{a}(41 \%)$ et $8 \mathrm{~b}(30 \%)$. La question 9 , qui demandait de faire une inférence pragmatique était également peu réussie (25\%). À la question 5a, 60\% d'entre eux ont été en mesure de comprendre un mot de substitution, mais pas celui de la $5 b$ $(19 \%)$.

En avril, les élèves étaient plus nombreux à identifier l'idée principale explicite de paragraphes (54\%), mais peu ont réussi lorsque l'idée était implicite (25\%). Ils ont en majorité réussi à identifier un référent à un mot de substitution en 5a (83\%), mais toujours difficilement en $5 \mathrm{~b}(33 \%)$. Plus des deux tiers des élèves ont réussi les questions portant sur les inférences lexicales (8a et 8b), pragmatiques (9) et logiques (10). La question sur la structure du texte (question 11) est maintenant réussie par $68 \%$ des élèves. La seule question qui est moins réussie qu'au pré-test est la question 7 , demandant de faire une inférence causale. Par contre, la question 6, qui exigeait une opération semblable, est réussie davantage ( $82 \%$ en avril, versus $65 \%$ en septembre).

Les taux de réussite des élèves de la $6^{e}$ année du groupe avec intervention

La majorité des élèves ont réussi le repérage d'information $(89 \%$ et $86 \%)$ ainsi que la question 5a portant sur un mot de substitution (76\%). Plus des deux tiers d'entre 
eux $(68 \%)$ ont correctement identifié les idées principales explicites et implicites ainsi qu'un mot de substitution à la question $5 \mathrm{~b}(67 \%)$.

Néanmoins, seulement $54 \%$ et $43 \%$ ont effectué les inférences causales attendues (questions 6 et 7), 30\% ont réussi à faire une inférence logique (question 10) et seulement $17 \%$ l'inférence lexicale de la question 8 b. Enfin, seulement $9 \%$ des élèves ont pu comprendre la structure du texte suffisamment pour compléter un schéma comprenant quatre à six éléments attendus sur six, et ce, toujours en septembre. En avril, $43 \%$ des élèves y sont arrivés.

Toutes les questions ont d'ailleurs été réussies par un plus grand nombre d'élèves en avril, mis à part la question 4, soit l'identification d'une idée principale implicite. Une majorité d'élèves réussissent les questions $1,2,3,5$ a et $5 b$, puis la 9 et la 10 . Près des $3 / 4$ d'entre eux réussissent maintenant les questions 6,7 et 8 .

\section{Différence entre les groupes avec et sans intervention en septembre}

En septembre, davantage d'élèves provenant des groupes sans intervention ont réussi à répondre à la majorité des questions des épreuves, et ce, en $4^{\mathrm{e}}, 5^{\mathrm{e}}$ et $6^{\mathrm{e}}$ année. Le groupe sans intervention est donc d'emblée plus performant.

En $4^{\mathrm{e}}$ année, les élèves du groupe avec intervention étaient ainsi beaucoup plus faibles que les élèves du groupe sans intervention en ce qui concerne la question 4 (24\% de réussite contre $82 \%)$ et les questions $5 \mathrm{a}(41 \%$ contre $77 \%)$ et $5 \mathrm{~b}(28 \%$ contre $48 \%)$.

En $5^{\mathrm{e}}$ année, les questions 3 et 4 étaient beaucoup moins réussies au prétest par les groupes avec intervention (respectivement $37 \%$ contre $59 \%$ et $20 \%$ contre $56 \%$ ). Il en va de même pour la question 8 b (30\% contre $63 \%)$ et $9(25 \%$ contre $81 \%)$.

En $6^{\mathrm{e}}$ année, les écarts les plus grands, toujours au prétest, se situaient surtout aux questions 7 (43\% contre 61\%), 8a (46\% contre 64\%), 9 (31\% contre $89 \%), 10(30 \%$ contre $67 \%$ ), puis la dernière question qui exigeait une compréhension de la structure globale du texte $(9 \%$ contre $56 \%)$.

\section{Différence entre les groupes avec et sans intervention en avril}

En avril, les élèves de $4^{\mathrm{e}}$ année du groupe avec intervention étaient plus nombreux à réussir les questions 6, 10 et 11 que les groupes sans intervention, alors que c'était l'inverse en septembre. On observe également ce phénomène pour les questions 2 et 3 en $5^{\mathrm{e}}$ année et pour les questions $3,6,8 \mathrm{a}, 8 \mathrm{~b}$ et 10 en $6^{\mathrm{e}}$ année. Lors de ce même posttest, les groupes sans intervention avaient des taux de réussite plus bas qu'en début d'année en $4^{\mathrm{e}}$ année pour les questions 4 et 11 , en $5^{\mathrm{e}}$ année pour les questions $2,3,8 \mathrm{a}, 9$ et en $6^{\mathrm{e}}$ année pour les questions $2,4,8 \mathrm{a}, 8 \mathrm{~b}, 10$ et 11 .

\section{Différences de moyenne de progrès entre les groupes}

Afin de voir si les groupes avec et sans intervention ont progressé entre le prétest et le posttest, des comparaisons de moyennes ont été effectuées. Le tableau 3 présente ainsi les moyennes au prétest, au posttest et la moyenne de progrès pour les groupes d'élèves de $4^{\mathrm{e}} 5^{\mathrm{e}}$ et $6^{\mathrm{e}}$ année avec et sans intervention. Rappelons que les épreuves en $4^{\mathrm{e}}$ année sont sur 16 points, puis sur 18 points en $5^{\mathrm{e}}$ et $6^{\mathrm{e}}$ année. 
Tableau 3

Moyennes au prétest, au posttest et moyenne de progrès pour les élèves de $4^{\mathrm{e}}, 5^{\mathrm{e}}$ et $6^{\mathrm{e}}$ année avec et sans intervention

\begin{tabular}{llllccc}
\hline Élèves & $\mathrm{N}$ & $\begin{array}{c}\mathrm{M} \\
\text { Pré }\end{array}$ & $\begin{array}{c}\mathrm{M} \\
\text { Post }\end{array}$ & $\begin{array}{c}\mathrm{M} \\
\text { Progrès }\end{array}$ & $\begin{array}{c}\text { ÉT } \\
\text { M Progrès }\end{array}$ & $\mathrm{F}$ \\
\hline $4^{\mathrm{e}}$ avec intervention & 110 & 6.8 & 11.15 & 4.34 & 3.59 & $17.47^{*}$ \\
$4^{\mathrm{e}}$ sans intervention & 36 & 9.54 & 11.19 & 1.61 & 2.75 & \\
$5^{\mathrm{e}}$ avec intervention & 89 & 9.1 & 12.13 & 2.98 & 3.39 & $16.55^{*}$ \\
$5^{\mathrm{e}}$ sans intervention & 30 & 12.34 & 12.83 & 0.23 & 2.58 & \\
$6^{\mathrm{e}}$ avec intervention & 74 & 8.6 & 12.75 & 4.08 & 2.92 & $22.05^{*}$ \\
$6^{\mathrm{e}}$ sans intervention & 35 & 11.69 & 12.65 & 1.03 & 3.64 & \\
\hline
\end{tabular}

$* \mathrm{p}<0.05$

$\mathrm{Au}$ prétest en septembre, les élèves des classes avec intervention de tous les degrés avaient des moyennes nettement inférieures que leurs pairs de l'école sans intervention. En $4^{\mathrm{e}}$ année, les élèves des écoles avec intervention avaient une moyenne de 6,8 sur 16 alors que les élèves des classes sans intervention obtenaient une moyenne de 9,54. Toujours au prétest, en $5^{\mathrm{e}}$ et en $6^{\mathrm{e}}$ année, la différence des moyennes entre les groupes était encore plus élevée, cette fois les épreuves valant 18 points.

Cette différence entre les moyennes s'est réduite en fin d'année scolaire. Les élèves des groupes avec intervention avaient des moyennes qui rejoignaient pratiquement celles des groupes sans intervention. En $6^{\mathrm{e}}$ année, le groupe avec intervention obtenait une moyenne d'ailleurs plus élevée en avril que les groupes sans intervention. Enfin, une analyse de variance a permis de constater que les élèves des classes avec intervention ont augmenté leur moyenne davantage, et ce, de façon significative.

\section{Discussion}

Bien que plusieurs études précédentes aient démontré l'intérêt de l'enseignement explicite des stratégies de compréhension, il demeurait difficile de proposer ce type d'enseignement, qui s'avérait nouveau et complexe, à des enseignants vivant déjà de grandes problématiques dans leur milieu. Il leur était également demandé d'intégrer davantage de textes courants authentiques provenant de diverses sources, ce qui suscitait l'utilisation et la projection de textes numériques à partir desquels la modélisation des stratégies était proposée. Les enseignants devaient donc utiliser d'autres textes que ceux venant des manuels scolaires habituels. L'approche présentée se voulait ainsi une occasion de formation continue pour les enseignants qui, malgré leur horaire déjà surchargé, souhaitaient aborder de nouvelles façons d'améliorer les compétences en lecture des élèves. Les résultats de cette étude doivent donc être interprétés en sachant que les enseignants participants ont reçu des journées de libération de leur tâche d'enseignement pour mieux s'intégrer au projet, de l'accompagnement individuel et de la modélisation en classe par une orthopédagogue et une conseillère pédagogique, un guide d'accompagnement, des fiches d'activités et une banque de textes courants préalablement 
sélectionnés à partir de sources diverses et parfois même adaptés pour soutenir leur enseignement.

Les élèves participants à l'étude étaient les plus faibles de la commission scolaire en ce qui a trait aux compétences en français. Cela s'est d'ailleurs reflété dès le prétest, car des élèves d'une autre école ayant le même indice de défavorisation et vivant également en banlieue se sont montrés plus performants pour la majorité des questions de l'épreuve et ont ainsi obtenu une moyenne nettement plus élevée. Néanmoins, entre le début et la fin d'année, les élèves participants à l'intervention ont grandement amélioré leurs résultats, ce qui n'a pas été le cas des élèves des classes sans intervention. À cet égard, bien qu'il puisse sembler plus difficile pour un groupe de s'améliorer grandement lorsqu'il performe déjà bien au prétest, il demeure que le groupe d'élèves sans intervention aurait pu augmenter sa moyenne de six points au moins (sur 16 en $4^{\mathrm{e}}$ année, puis sur 18 en $5^{\mathrm{e}}$ et $6^{\mathrm{e}}$ ) entre le début de l'année et la fin. Les moyennes de progrès sont à leurs égards très faibles et il est nécessaire de se questionner sur les apprentissages relatifs aux stratégies de compréhension réalisées en salle de classe qui utilisent des approches et du matériel plus typiques. Le groupe avec intervention a d'ailleurs pratiquement rattrapé l'écart qui le séparait du groupe sans intervention en ce qui concerne les élèves de la $4^{\mathrm{e}}$ et de la $5^{\mathrm{e}}$ année. Les élèves de la $6^{\mathrm{e}}$ année, quant à eux, ont non seulement rattrapé cet écart, mais ils ont performé davantage que le groupe sans intervention.

D'ailleurs, les questions davantage réussies en fin d'année scolaire par les élèves des groupes avec intervention sont pratiquement toutes liées à une compréhension de haut niveau de textes courants, ce qui était grandement travaillé dans ce projet. Cela réaffirme des constats abordés au début de cet article concernant l'importance de développer des stratégies de compréhension de lecture appropriées à l'égard des textes courants. Ces textes sont omniprésents à l'école, dans les loisirs et dans le monde professionnel (Hughes-Hassell \& Rodge, 2007; Kamil \& Lane, 1998; Moss, 2004). Auprès d'élèves présentant des risques d'éprouver des difficultés, les stratégies de compréhension à l'égard de ces textes sont importantes à enseigner dès le primaire (Bryce, 2011; Hall \& Sabey, 2007; Juel, Hebard, Park-Haubner \& Moran, 2010), sans oublier la poursuite de cet enseignement sur une longue période de temps (Sweet \& Snow, 2003) afin de former des lecteurs compétents et autonomes.

En ce qui a trait plus précisément aux textes offerts, rappelons que dans toute classe du primaire au Québec, l'enseignant aborde les sciences, l'univers social et l'éthique et la culture religieuse, des disciplines utilisant toutes une majorité de textes courants. Les élèves sont donc en contact avec ces types de textes pratiquement au quotidien. Il est alors nécessaire de se demander dans quelle mesure ces textes, provenant souvent de manuels scolaires, sont intéressants et pertinents sur le plan de leur structure, de leur vocabulaire et de leurs sujets pour former des lecteurs polyvalents à long terme. Il est également pertinent de se questionner à l'égard de l'enseignement des stratégies de lecture à l'aide de ces textes qui, bien qu'ils soient abordés dans d'autres disciplines, méritent d'être lus avec tout autant d'efforts de compréhension que ceux lus dans les périodes d'enseignement du français. Dans le cadre du projet, non seulement les élèves ont abordés des textes courants choisis en fonction de leurs structures, de leurs contenus et de leurs supports (parfois imprimés, parfois numériques), mais on leur a aussi offert un 
enseignement explicite de stratégies de compréhension à leur égard. Il est donc possible de croire que dans ces classes avec intervention, l'offre de soutien et l'ajout de textes courants pertinents et diversifiés portant sur des sujets intéressants aient contribué à faire progresser les élèves.

De surcroît, certains résultats de ce projet méritent une attention particulière. En fin de $6^{\mathrm{e}}$ année, moins d'élèves des classes avec intervention ont réussi la question portant sur l'identification de l'idée principale implicite qu'en début d'année. Pourtant, un plus grand nombre d'élèves sont arrivés à identifier l'idée principale explicite. Après avoir demandé l'avis des enseignants à cet égard, ces derniers nous ont confié avoir enseigné la stratégie d'identification des idées principales explicites sans avoir mis autant d'énergie sur l'implicite. Plusieurs raisons ont été données: le manque de temps, l'impression de répéter une activité, l'incompréhension de cette stratégie. Cela a permis de comprendre une limite relative à l'enseignement explicite des stratégies. En effet, les enseignants ont montré aux élèves comment identifier l'idée principale en se centrant uniquement, et de façon erronée, sur le fait que cette dernière soit toujours explicite. Or, l'idée principale de chaque paragraphe d'un texte peut être explicite, implicite ou même ambigüe. Cette nuance doit être enseignée par les enseignants et doit faire l'objet de pratiques à l'intérieur de textes variés. Comme ce ne fut visiblement pas le cas, en avril, les élèves se sont donc efforcés de trouver une phrase présente dans chaque paragraphe lorsqu'ils ont eu à identifier une idée principale, même lorsque l'idée principale était implicite et qu'elle devait être formulée. Cela nous mène à préciser l'importance pour chaque enseignant de connaitre et de comprendre lui-même les stratégies à enseigner ainsi que les effets que peuvent avoir la décision d'enseigner une stratégie de manière partielle, dans un contexte limité ou sans aborder plusieurs exemples et contextes pour la mettre en pratique.

\section{Implications pédagogiques}

À l'égard des praticiens, il est important de retenir que la lecture de textes intéressants et venant de diverses sources ainsi que l'enseignement de type explicite des stratégies en quatre étapes, qui implique un étayage décroissant envers l'élève au fur et à mesure que ce dernier gagne en autonomie, sont des éléments qui peuvent favoriser le progrès de lecteurs démontrant des niveaux de compétences différents. Même si ce projet se voulait très structurant pour les enseignants autant en ce qui avait trait aux stratégies à enseigner que du calendrier à respecter, il offrait des textes courants provenant de sources diverses afin d'enseigner ces stratégies à partir de textes authentiques, rédigés par des auteurs de magazines, de livres et de sites Internet avec l'intention d'informer ou encore de divertir un jeune public. L'approche présentée s'est donc révélée intéressante pour le milieu ciblé lors du projet et pourrait avoir un certain potentiel pour un milieu analogue.

Tels que décrits dans cette discussion, plusieurs éléments de ce projet ont peut-être facilité l'appropriation d'une nouvelle approche d'enseignement par les enseignants. Le fait également de participer à un projet commun a créé une synergie entre les enseignants de différents degrés qui partageaient un calendrier pour introduire les stratégies.

Enfin, les épreuves conçues dans ce projet ont également joué un rôle formateur. Afin que les enseignants comprennent les progrès de leurs élèves, ils ont dû s'approprier le modèle d'Irwin (2006) qui était à la base de la création de ces épreuves. En ce qui a 
trait à la formation initiale et continue, les épreuves de compréhension de lecture peuvent représenter du matériel à critiquer et à approfondir. Ces épreuves pourraient aussi être utiles pour cibler des difficultés précises, car elles comprennent des questions ayant des objectifs définis et appuyés sur un modèle théorique multidimensionnel de la compréhension écrite.

\section{Implications pour la recherche}

Il serait intéressant de suivre les élèves de façon longitudinale et de voir si les progrès réalisés entre le début et la fin de l'année scolaire se poursuivent jusqu'au secondaire. Il faudrait alors détenir des épreuves pour continuer de mesurer ainsi leurs progrès. Le contexte de cette recherche est sans doute exceptionnel pour le milieu et difficile à reproduire dans toutes les écoles. À cet égard, il est essentiel d'étudier comment une telle approche peut être adoptée dans les écoles sans autant de soutien, sachant que le roulement de personnel est un élément perturbant souvent la mise en place de projets novateurs ou unificateurs. Des questions demeurent ainsi en suspens et mériteraient d'être approfondies. Un guide d'accompagnement serait-il suffisant pour que les enseignants s'approprient une telle approche ? Des modélisations à partir de vidéos pourraient-elles soutenir suffisamment les enseignants qui s'intéressent à l'enseignement explicite de telles stratégies ? De telles modélisations pourraient-elles être bénéfiques également pour les élèves ? Un système de mentorat pourrait-il convenir lors de l'arrivée de nouveaux enseignants dans une école qui a déjà adopté une telle approche commune entre les degrés scolaires?

\section{Limites de l'étude}

Les deux groupes, avec et sans interventions, se sont révélés non équivalents dès le départ. Actuellement, il est donc impossible de comprendre comment deux groupes d'élèves identiques progressent dépendamment de leur participation à un tel projet. Par contre, il a été possible de voir comment le groupe avec intervention peut rattraper ou dépasser un groupe plus fort. Il est aussi concevable qu'une telle approche centrée sur l'enseignement explicite favorise beaucoup plus les élèves plus faibles, ce qui expliquerait les différences de progrès marquantes entre les groupes. Enfin, parce que les textes courants sont omniprésents, l'analyse des facteurs scolaires ayant un effet positif sur la compréhension de ce type d'écrits demeure très complexe. En effet, au fur et à mesure que les élèves vieillissent, ils accumulent des expériences scolaires diverses à l'égard de l'apprentissage de la lecture, ils sont exposés quotidiennement à des textes variés et vivent d'autres expériences de lecture informelles à l'école, à la maison et dans leurs activités parascolaires.

\section{Remerciements}

Cette recherche a été rendue possible grâce à une subvention du Ministère de l'éducation, du Loisir et du Sport (MELS) du Québec. 


\section{Références}

Aikens, N. L. \& Barbarin, O. (2008). Socioeconomic differences in reading trajectories: The contribution of family, neighborhood, and school contexts. Journal of Educational Psychology, 100(2),235-251.

Archer, A. \& Hughes, C. (2011). Explicit Instruction: Effective and Efficient Teaching. New York, NY: The Guilford Press.

Berkeley, S., Scruggs, T. E. \& Mastropieri, M. A. (2010). Reading comprehension instruction for students with learning disabilities, 1995-2006: A meta-analysis. Remedial and Special Education, 31(6),423-436.

Biancarosa, G. \& Snow, C. E. (2004). Reading Next-A Vision for Action and Research in Middle and High School Literacy: A Report to Carnegie Corporation of New York. Washington, DC: Alliance for Excellent Education. Récupéré le 28 février 2010 à : http://www.all4ed.org/files/ReadingNext.pdf

Bissonnette, S., Richard, M., Gauthier C. \& Bouchard, C. (2010). Quelles sont les stratégies d'enseignement efficaces favorisant les apprentissages fondamentaux auprès des élèves en difficulté de niveau élémentaire? Résultats d'une mégaanalyse. Revue de recherche appliquée sur l'apprentissage, 3, 1-35.

Bråten, I. \& Anmarkrud, Ø. (2013), Does naturally occurring comprehension strategies instruction make a difference when students read expository text? Journal of Research in Reading, 36, 42-57. doi: 10.1111/j.1467-9817.2011.01489.x

Bryce, N. (2011).Meeting the reading challenges of science textbooks in the primary grades. The Reading Teacher, 64(7), 474-485. doi:10.1598/RT.64.7.1

Cervetti, G., Pearson, P. D., Barber, J., Hiebert, E. \& Bravo, M. (2007). Integrating literacy and science: The research we have, the research we need. Dans M. Pressley, A. K. Billman, K. Perry, K. Refitt \& J. Reynolds (Dir.), Shaping literacy achievement (pp. 157-174). New York, NY: The Guilford Press.

Chall, J., Jacobs, V. \& Baldwin, L. (1990). The reading crisis: Why poor children fall behind. Cambridge, MA: Harvard University Press.

Coley, R. (2002). An uneven start: Indicators of inequality in school readiness. Princeton, NJ: Educational Testing Service.

Conseil Canadien sur l'Apprentissage (2008). Lire l'avenir : Pour répondre aux besoins futurs du Canada en matière de littératie. Repéré le 9 septembre 2012 à : http://www.ccl-cca.ca/CCL/Reports/ReadingFuture/ReadingFutureReport-2.html

Desrosiers, H. \& Tétrault, K. (2012). Les facteurs liés à la réussite aux épreuves obligatoires de français en sixième année du primaire : un tour d'horizon. Étude longitudinale du développement des enfants du Québec (ÉLDEQ 1998-2010) -De la naissance à 12 ans. Institut de la statistique du Québec, 7(1), 1-40.

Donovan, C. A. \& Smolkin, L. S., (2006). Children's understanding of genre and writing development. Dans C.A. MacArthur, S. Graham, \& J. Fitzgerald (Dir.), Handbook of Writing Research (pp. 131-143). New York, NY: The Guilford Press.

Duke, N. K. (2000). 3.6 minutes per day: The scarcity of informational texts in first grade. Reading Research Quarterly, 35(2), 202-224.

Dymock, S. (2005), Teaching Expository Text Structure Awareness. The Reading Teacher, 59(2), 177-181. doi: 10.1598/RT.59.2.7 
Fisher, D., Frey, N. \& Lapp, D. (2012). Text complexity: Raising rigor in reading. Newark, DE: International Reading Association.

Gajria, M., Jitendra, A. K., Sood, S., \& Sacks, G. (2007). Improving comprehension of expository text in students with LD: A research synthesis. Journal of Learning Disabilities, 40(3), 210- 225.

Gersten, R., Fuchs, L. S., Williams, J. P., \& Baker, S. (2001). Teaching reading comprehension strategies to students with learning disabilities: A review of research. Review of Educational Research, 71(2), 279-320.

Goldschmidt, P. (2010). Evaluation of Seeds of Science/Roots of Reading: Effective tools for developing literacy through science in the early grades. Los Angeles, CA: University of California, National Center for Research on Evaluation, Standards, and Student Testing (CRESST).

Gunning, T. (2003). The Role of Readability in Today's Classrooms. Topics in Language Disorders, 23(3), 175-189.

Guthrie, J. T., \& Davis, M. H. (2003). Motivating struggling readers in middle school through an engagement model of classroom practice. Reading \& Writing Quarterly, 19(1), 59-85.

Hall, K.M., \& Sabey, B.L. (2007). Focus on the facts: Using informational texts effectively in early elementary classrooms. Early Childhood Education Journal, 35(3), 261-268.

Hart, B. \& Risley, T.R. (2004). The Early Catastrophe. Education Review, 77 (1), 100118.

Hill, K.D. (2014). A second grade teacher's innovations toward multimodal literacies in an urban primary school. Language \& Literacy: A Canadian Educational EJournal,16 (1), 21-33.

Hughes-Hassell, S. \& Rodge, P. (2007). The leisure reading habits of urban adolescents. Journal of Adolescent \& Adult Literacy, 51(1), 22-33.

Irwin, J. (2006). Teaching reading comprehension processes (3e éd.). Boston, MA: Allyn \& Bacon.

Juel, C., Hebard, H., Park-Haubner, J., \& Moran, M. (2010). Reading through a disciplinary lens. Educational Leadership, 67, 13-17.

Juel, C. \& Minden-Cupp, C. (2000). Learning to read words: Linguistic units and instructional strategies. Reading Research Quarterly, 35(4), 458-492.

Kamil, M. \& Lane, D. (1998). Researching the relation between technology and literacy: An agenda for the 21st century. Dans D. Reinking, M.C. McKenna, L.D. Labbo, \& R.D. Kieffer (Dir.), Handbook of literacy and technology: Transformations in a post-typographic world (pp. 323-341). Mahwah, NJ: Erlbaum.

Leu, D.J., Kinzer, C.K., Coiro, J., Castek, J. \& Henry, L. (2013). New literacies : A duallevel theory of the changing nature of literacy, instruction and assessment. Dans R. B. Ruddell \& N.J. Unrau (Dir.) Theoretical models and processes of reading (5th ed., pp. 1570-1613). Newark, DE : International Reading Association.

Martel, V., Lévesque, J.-Y. \& Aubin-Horth, S. (2012). Compréhension en lecture au primaire : actualisation des pratiques enseignantes. Nouveaux c@hiers de la recherche en éducation, 15 (1), 87-106. 
McRae, A. \& Guthrie, J. T. (2009). Promoting reasons for reading: Teacher practices that impact motivation. Dans E. H. Hiebert (Ed.), Reading more, reading better (pp. 55-76). New York, NY : The Guilford Press.

Ministère de l'éducation, du loisir et du sport (MELS). (2011). Indices de défavorisation par école. Gouvernement du Québec.

Mesnager,J. (2002). Pour une étude de la difficulté des textes, ou la lisibilité revisitée. Le Français aujourd'hui, 137, 29-42.

Mesnager, J. (2011). Le vocabulaire et son enseignement. Évaluation de la difficulté des textes. Eduscol. Texte repéré ici : www.eduscol.education.fr/vocabulaire

Meyer, B. \& Ray, F. (2011). Structure strategy interventions: Increasing reading comprehension of expository text. International Electronic Journal of Elementary Education, 4(1), 127-152.

Moss, B. (2004). Teaching expository text structures through information trade book retellings. The Reading Teacher, 57(8), 710-718.

Ness, M. K. (2011). Teachers' Use of and Attitudes toward Informational Text in K-5 Classrooms. Reading Psychology, 32(1), 28-53.

Orr, A. J. (2003). Black-White differences in achievement: The importance of wealth. Sociology of Education, 76(4), 281-304.

Palincsar, A. S. \& Brown, A. L. (1985). Reciprocal teaching: Activities to promote "reading with your mind." Dans T. L. Harris \& E. J. Cooper (Dir.) Reading, Thinking, and Concept Development: Strategies for the Classroom (pp.147-159). New York, NY: The College Board.

Paris, S. G. (2005). Reinterpreting the development of reading skills. Reading Research Quarterly, 40(2), 184-202.

Pentimonti, J.M., Zucker, T.A., Justice, L.M. \& Kaderavek, J.N. (2010). Informational Text Use in Preschool Classroom Read-Alouds. The Reading Teacher, 63(8), 656-665.

Perfetti , C. , Landi , N. \& Oakhill , J. (2005). The acquisition of reading comprehension skill. Dans M.J. Snowling \& C. Hulme (Dir.), The science of reading: A handbook (pp. 227 - 247). Oxford : Blackwell.

Pressley, M. (2006). Reading instruction that works. The case for balanced teaching (3e éd.). New York, NY: The Guilford Press.

Ritchey, K. D., Silverman, R. D., Montanaro, E. A., Speece, D. L. \& Schatschneider, C. (2012). Effects of a tier 2 supplemental reading intervention for at-risk fourthgrade students. Exceptional Children, 78(3), 318-334.

Rosenshine, B. \& Meister, C. (1993). Reciprocal teaching: A review of 19 experimental studies. Urbana, IL: Center for the Study of Reading.

Sáenz, L. M. \& Fuchs, L. S. (2002). Examining the reading difficulty of secondary students with learning disabilities: Expository versus narrative text. Remedial and Special Education, 23(1), 31-41.

Scharlach, T.D. (2008). START comprehending: Students and teachers actively reading text. The Reading Teacher, 62(1), 20-31.

Sencibaugh, J. M. (2007). Meta-analysis of reading comprehension interventions for students with learning disabilities: Strategies and implications. Reading Improvement, 44(1), 6-22. 
Shanahan T. \& Shanahan, C. (2008). Teaching disciplinary literacy to adolescents. Harvard Educational Review, 78, 40-59.

Snow, C. (2008, March). Why supporting language development is the best preparation for literacy success. Paper presented at The 40th Banff International Conference on Behavioural Science, Banff, AB.

Sweet, A. \& Snow, C. (2003). Rethinking reading comprehension :Solving problems in the teaching of literacy. New York, NY: The Guilford Press.

Torgesen, J. K. \& Houston, D. D., Rissman, L. M., Decker, S. M., Roberts, G., Vaughn, S., Wexler, J., ... Lesaux, N. (2007). Academic literacy instruction for adolescents: A guidance document from the Center on Instruction. Portsmouth, NH: RMC Research Corporation, Center on Instruction. Repéré le 10 février 2011 à: ttp://www.centeroninstruction.org/files/Academic\%20Literacy.pdf

Turcotte, C. \& Cloutier, E. (2014). Le rappel stimulé pour mieux comprendre les stratégies de lecture d'élèves du primaire à risque et compétents. La revue Canadienne d'éducation, 37(1), 72-95

Van Dijk, T. A. \& Kintsch, W. (1983). Strategies of discourse comprehension. New York, NY: Academic Press.

Watson, S. M. R., Gable, R. A., Gear, S. B. \& Hughes, K. C. (2012). Evidence-Based Strategies for Improving the Reading Comprehension of Secondary Students: Implications for Students with Learning Disabilities. Learning Disabilities Research \& Practice, 27(2), 79-89. doi: 10.1111/j.1540-5826.2012.00353.x

Wigent, C.A. (2013). High school readers: A profile of above average readers and readers with learning disabilities reading expository text. Learning and Individual Differences 24, 134-40.

Woolley, G. (2011). Reading Comprehension: Assisting Children with Learning Difficulties. Dordrecht, The Netherlands: Springer International.

Worthy, J., Moorman, M. \& Turner, M. (1999). What Johnny Likes to Read Is Hard to Find in School. Reading Research Quarterly, 34(1), 12-27. doi: 10.1598/RRQ.34.1.2

Biographie des auteures

Catherine Turcotte is a professor at the Université du Québec à Montréal. She is interested in reading comprehension difficulties among primary grade students. / Catherine Turcotte est professeure à l'Université du Québec à Montréal. Elle s'intéresse aux difficultés en compréhension de lecture des élèves du primaire.

Marie-Hélène Giguère is a doctoral student at the Université de Montréal. She is interested in teaching and learning grammar and reading in primary and secondary schools. She also works as a pedagogical adviser in a Québec's school board. / MarieHélène Giguère est doctorante à l'Université de Montréal. Elle s'intéresse à l'enseignement et à l'apprentissage de la grammaire et de la lecture au primaire et au secondaire. Elle travaille également comme conseillère pédagogique dans une commission scolaire au Québec. 
Marie-Julie Godbout is a special education teacher and a research agent at the Université du Québec à Montréal. She is interested in children experiencing learning difficulties, in exemplary instruction and in evaluation of reading comprehension. / Marie-Julie Godbout est orthopédagogue et agente de recherche à 1'Université du Québec à Montréal. Elle s'intéresse aux élèves éprouvant des difficultés d'apprentissage, aux interventions efficaces et à l'évaluation de la compréhension écrite. 